\title{
$\mathbb{A}$ Economics Bulletin
}

Volume 37, Issue 3

\section{Investment in education under disappointment aversion}

\author{
Dan Anderberg \\ Royal Holloway University of London
}

\author{
Claudia Cerrone \\ Max Planck Institute for Research on Collective Goods
}

\begin{abstract}
This paper develops a model of risky investment in education under disappointment aversion, modelled as loss aversion around one's endogenous expectation. The model shows that disappointment aversion reduces investments in education for lower ability people and increases investments for higher ability people, thereby magnifying the gap between them generated by the riskiness of education.
\end{abstract}

Thanks to Benjamin Bachi, Olga Chiappinelli, Christoph Engel, Svenja Hippel and Ester Manna. Financial support by the Nuffield Foundation (grant EDU/41689) is gratefully acknowledged. An earlier and substantially different version of this paper circulated under the title 'Education, disappointment and optimal policy".

Citation: Dan Anderberg and Claudia Cerrone, (2017) "Investment in education under disappointment aversion", Economics Bulletin, Volume 37, Issue 3, pages 1533-1540

Contact: Dan Anderberg - dan.anderberg@rhul.ac.uk, Claudia Cerrone - cerrone@coll.mpg.de.

Submitted: December 17, 2016. Published: July 02, 2017. 


\section{Introduction}

Dropping out of education at an early age has negative consequences on future earnings and non-pecuniary dimensions such as crime, health and teenage pregnancy (Oreopoulos \& Salvanes 2011). There is no single explanation for early school dropout: previous literature has explored the role played by inaccurate perception of returns to schooling (Jensen 2010), impatience (Cadena \& Keys 2015) and other "behavioural barriers" (Lavecchia et al. 2014). Closely related to early school-leaving is the observation that empirical rates of return on education are often quite high - higher than returns on physical capital, and particularly for individuals from disadvantaged families. ${ }^{1}$

This paper considers another possible explanation for early school-leaving and high remaining marginal rates of return on education: potential disappointment at doing worse than expected. Evidence shows that people's decision to stay in school is affected by their expectations (Goux et al. 2014). It is reasonable to think that less academically talented people may drop out of school early in order to avoid raising their expectations, thereby risking disappointment. In this paper, we develop a model of risky investment in education under disappointment aversion, where disappointment aversion is defined as loss aversion around the agent's expected outcome, and the expected outcome depends on the investment level chosen by the agent and on her ability. The model is used to explore the impact of disappointment aversion on investments in education. In particular, we highlight how disappointment aversion creates wider heterogeneity in educational choices and rates of return across ability types. ${ }^{2}$

\section{The model}

Set-up There are two periods: the "present" or "youth", and the "future" or "adulthood". The individual devotes a fraction $\lambda \in[0,1]$ of the first period to investing in human capital (acquiring education). The remaining fraction of the first period, $1-\lambda$, is spent working for a wage, $w_{0}>0$. The cost of investing in education is thus given by foregone earnings. All consumption occurs in the second period, hence the individual's earnings in youth are carried forward to adulthood in the form of savings, $s(\lambda) \equiv(1-\lambda) w_{0}$. For simplicity, we ignore interest on savings, hence one unit of savings carried forward increases adult consumption by one unit.

In the second period, the individual works full time, and consumes her earnings and savings. ${ }^{3}$ Preferences over consumption, $v$, are strictly increasing, strictly concave, twice differentiable and the third derivative equals zero. The individual will obtain one of two potential wages $\left\{w_{L}, w_{H}\right\}$, where $w_{H}>w_{L} \geq w_{0}$. The probability of wage $w_{j}$, denoted $\pi^{j}(\lambda, \alpha) \equiv \operatorname{Pr}\left(w_{j} \mid \lambda, \alpha\right)$, depends on the individual's investment, $\lambda$, and on her academic ability, $\alpha$, where $\alpha \in[\underline{\alpha}, \bar{\alpha}]$. It is assumed that an increase in either education or ability increases the probability of a high wage but at a decreasing rate, and that education and ability are complements.

Assumption 1. $\pi^{H}(\lambda, \alpha)$ is a continuous function with $\pi_{\lambda}^{H}(\lambda, \alpha)>0, \pi_{\lambda \lambda}^{H}(\lambda, \alpha)<0, \pi_{\alpha}^{H}(\lambda, \alpha)>$ $0, \pi_{\alpha \alpha}^{H}(\lambda, \alpha) \leqslant 0$ and $\pi_{\lambda \alpha}^{H}(\lambda, \alpha)>0$ for all $\alpha$ and $\lambda$. Furthermore, $\pi^{H}(0, \alpha)=0$ and

\footnotetext{
${ }^{1}$ See Carneiro \& Heckman (2002) for a detailed critical discussion of these "stylized" facts.

${ }^{2}$ Lecouteux \& Moulin (2015) show that, when students' reference points are given by their origins (i.e. whether they are from a poorer or wealthier background), the optimal policy implies lower tuition fees for poorer students.

${ }^{3}$ See Section 4 for a discussion about a labour supply choice in adulthood.
} 
$\lim _{\lambda \rightarrow 0} \pi_{\lambda}^{H}(\lambda, \alpha)=+\infty$ and $\lim _{\lambda \rightarrow 1} \pi_{\lambda}^{H}(\lambda, \alpha)=0$ for all $\alpha$.

The timing is as follows. In the first period, the individual learns her ability and chooses a level of investment in education $\lambda{ }^{4}$ In the second period, her wage realization is revealed; she then works full time and consumes her earnings and savings.

Disappointment Aversion In disappointment aversion models (see Bell 1985, Loomes \& Sugden 1986, Koszegi \& Rabin 2006, Koszegi \& Rabin 2007), the agent is sensitive to deviations from what she expected to receive. In particular, she is loss averse around her expectation, which implies that losses relative to her expectation are more painful than equal-sized gains are pleasurable. We model disappointment aversion as loss aversion around the agent's expected utility, which depends on the investment level actually chosen by the agent. Hence, as in Koszegi \& Rabin (2007), the expectation-based reference point is choice-acclimating: it adjusts to the agent's choice.

Let $\bar{v}$ denote the expected utility of an individual with ability $\alpha$ who has made the investment $\lambda$. Then,

$$
\bar{v}(\lambda, \alpha) \equiv \sum_{j=L, H} \pi^{j}(\lambda, \alpha) v\left(w_{j}+(1-\lambda) w_{0}\right)
$$

The individual's reference point is given by (1). Disappointment aversion implies that the individual suffers a utility loss when failing to reach her expectation that is larger than the utility gain from exceeding her expectation. This can be formalised by assuming that the individual's "experienced" utility in the low wage and in the high wage states are $v^{L}+\psi^{L}\left(v^{L}-\bar{v}\right)$ and $v^{H}+\psi^{H}\left(v^{H}-\bar{v}\right)$ respectively, where $\psi^{L} \geq \psi^{H} \geq 0$, and $v^{L}$ and $v^{H}$ are shorthands for the indirect utility at the low wage and high wage. Taking the expectation over the wage realization yields the individual's ex-ante utility inclusive of disappointment aversion,

$$
V(\lambda, \alpha)=\sum_{j=L, H} \pi^{j}(\lambda, \alpha)\left(v^{j}+\psi^{j}\left(v^{j}-\bar{v}(\lambda, \alpha)\right)\right)
$$

Substituting for the expected value using (1) and rearranging yields,

$$
V(\lambda, \alpha)=\sum_{j=L, H} \pi^{j}(\lambda, \alpha) v^{j}-\psi \sigma(\lambda, \alpha) \Delta v
$$

where $\psi \equiv \psi^{L}-\psi^{H}, \Delta v \equiv v^{H}-v^{L}$ and $\sigma(\lambda, \alpha) \equiv \pi^{L}(\lambda, \alpha) \pi^{H}(\lambda, \alpha) .^{5}$

It should be noted that the assumptions made above on $v(\cdot)$ and $\pi^{H}(\cdot)$ are generally not sufficient to ensure that $V(\cdot)$ is globally concave in $\lambda{ }^{6}$ Indeed, $V(\lambda, \alpha)$ may be convex if both $v(\cdot)$ and $\pi^{H}(\cdot)$ are close to being linear. Fortunately, sufficient concavity of $\pi^{H}(\cdot)$ with respect to $\lambda$ will ensure that $V(\lambda, \alpha)$ is strictly concave even in the case of limited risk aversion. In order to avoid an overly discursive analysis of sufficient conditions for concavity, we will here simply assume that $V(\lambda, \alpha)$ is continuously differentiable and, due to the concavity of $\pi^{H}(\cdot)$,

\footnotetext{
${ }^{4}$ See Section 4 for a discussion about the assumption that $\alpha$ is perfectly known to the agent.

${ }^{5} \mathrm{~A}$ natural restriction on $\psi$ is that it does not exceed unity. This upper bound ensures that an agent with a sufficiently low success probability will always benefit from an increase in the success probability, i.e. will never prefer her success probability to be reduced even further.

${ }^{6}$ We are grateful to a referee for alerting us to a key source of non-concavity.
} 
strictly concave in $\lambda$ for any $\alpha \in[\underline{\alpha}, \bar{\alpha}] .^{7}$

The final term in (3), that captures disappointment aversion, has three components: (i) the strength of the aversion towards disappointment $\psi$; (ii) the utility gap between the good and the bad outcome $\Delta v$; (iii) the product of the probabilities $\sigma(\lambda, \alpha)$. For any $\alpha, \sigma(\lambda, \alpha)$ is an increasing function of $\lambda$ up to the point where $\pi^{H}(\lambda, \alpha)=1 / 2$ and decreasing in $\lambda$ thereafter. Note that with two potential wages, the wage variance is proportional to $\sigma(\lambda, \alpha)$. Hence, consistent with empirical evidence (Chen 2008) ${ }^{8}$, the model features an inverted U-shaped relation between $\lambda$ and wage risk at the individual level, although for low ability agents wage risk monotonically increases in investment in so far as for them $\pi^{H}(\lambda, \alpha)<1 / 2$ for any $\lambda \in[0,1]$.

\section{Educational investment behaviour}

In youth the individual chooses $\lambda^{*}(\alpha)$ to maximize (3), leading to the first order condition

$$
V_{\lambda}=\left(\pi_{\lambda}^{H}\left(\lambda^{*}(\alpha), \alpha\right)-\psi \sigma_{\lambda}\left(\lambda^{*}(\alpha), \alpha\right)\right) \Delta v-w_{0}\left\{E\left[v^{\prime}\right]-\psi \sigma\left(\lambda^{*}(\alpha), \alpha\right) \Delta v^{\prime}\right\}=0 .
$$

The unique solution, denoted $\lambda^{*}(\alpha)$, is, by the theorem of the maximum, a continuous function of $\alpha$. Due to the complementarity between investment and ability in the success probability, it is easy to see that $\lambda^{*}(\alpha)$ and $\pi^{*}(\alpha) \equiv \pi^{H}\left(\lambda^{*}(\alpha), \alpha\right)$ are strictly increasing in $\alpha .{ }^{9}$

Our interest is in showing how disappointment aversion can affect the investments of different types in opposite direction. To that end, we assume that there is sufficient heterogeneity in abilities to ensure that, in equilibrium, for some low types getting a low wage is more likely than getting a high wage, and, vice versa, for some high types getting a high wage is more likely than getting a low wage. ${ }^{10}$

Assumption 2. $\pi^{*}(\underline{\alpha})<1 / 2<\pi^{*}(\bar{\alpha})$.

Let $\widehat{\alpha}$ denote the ability type who chooses a level of education that induces equal probability of success and failure, i.e. $\pi^{*}(\widehat{\alpha})=1 / 2$. Assumption 2 implies that $\underline{\alpha}<\widehat{\alpha}<\bar{\alpha}$. Note that $\widehat{\alpha}$ is unique due to the monotonicity of the success probability in ability.

The rate of return on education is defined as the expected increase in adulthood earnings from a marginal increase in investment in education relative to the loss in youth earnings.

$$
\rho^{*}(\alpha) \equiv \frac{\pi_{\lambda}^{H}\left(\lambda^{*}(\alpha), \alpha\right) \Delta w}{w_{0}} .
$$

\footnotetext{
${ }^{7}$ It should also be noted that, since we have a compact choice space $\lambda \in[0,1]$, an optimal choice will always exist, and it is easy to provide examples showing that the implications of disappointment aversion continue to apply also in cases where $V(\cdot)$ fails to be concave in $\lambda$ over the entire unit interval.

${ }^{8}$ Chen (2008) shows that the decision to enter college in particular is associated with an increase in the level of earning risk at the individual level.

${ }^{9}$ One can show that the Inada conditions in Assumption 1 guarantee an interior solution for every ability type.

${ }^{10}$ Assumption 2 is not an assumption stated in terms of the primitives of the model. One could augment the Inada conditions in Assumption 1 to generate sufficient conditions on the primitives, e.g. $\pi^{H}(1, \underline{\alpha})<0.5$ would be sufficient to ensure that the lowest type makes a strictly positive investment but cannot attain a success probability above 0.5. However, such assumptions would be unnecessarily restrictive. Note for instance that it is not satisfied by the numerical example below.
} 


\subsection{Benchmark case}

Consider first the case with no disappointment aversion. The individual is choosing between two alternative investments: investment in human capital (education) and investment in physical capital (savings). Following Levhari \& Weiss (1974), the former is risky while the latter is safe, ${ }^{11}$ which causes the following deviation between their equilibrium returns: $\rho^{*}(\alpha)>1$ if a marginal increase in education increases wage risk and $\rho^{*}(\alpha)<1$ otherwise. In particular, in our model the latter implies that $\rho^{*}(\alpha)>1$ for lower ability types and $\rho^{*}(\alpha)<1$ for higher ability types, as wage risk increases with investment up to the point where the success probability is $1 / 2$ and decreases thereafter. This result is stated by Proposition 1.

Proposition 1. In the absence of disappointment aversion, $\rho^{*}(\alpha)>1$ for any $\alpha<\widehat{\alpha}$ and $\rho^{*}(\alpha) \leq 1$ otherwise.

\subsection{Disappointment aversion}

The introduction of disappointment aversion will differentially affect the chosen investments in education by low and high ability individuals. Low ability individuals respond by reducing their investments, while high ability individuals increase theirs. Proposition 2 demonstrates this result for the case of limited risk aversion. By limited risk aversion we mean that that the difference in marginal utility between the low and the high outcome, $\Delta v^{\prime}$, is arbitrarily small.

Proposition 2. Suppose that there is positive but limited risk aversion. Then there exists a unique type $\widetilde{\alpha} \in(\widehat{\alpha}, \bar{\alpha})$ such that disappointment aversion reduces the investment in education for ability types below $\widetilde{\alpha}$ and increases it for ability types above $\widetilde{\alpha}$.

The intuition is that less talented agents are discouraged from increasing their investment, as doing so would raise their expectations and hence the potential disappointment associated with a negative outcome. Conversely, more talented agents are encouraged to increase their investment in order to secure a favourable outcome and avoid potential disappointment. Both higher and lower ability types respond to disappointment averse preferences by adjusting their investment towards certainty. It is important to point out that limited risk aversion is a sufficient, but far from necessary condition, as the example below will show. ${ }^{12}$ Indeed, the example shows that, with an empirically relevant level of risk aversion (relative risk aversion lies between 0.5 and 1 over the relevant range of consumption), the deviation between the rate of return on education and unity in Proposition 1 is modest. Disappointment aversion, however, strongly reinforces the "Levhari-Weiss effect" in Proposition 1, significantly widening the dispersion of investments and hence of equilibrium rates of return.

\footnotetext{
${ }^{11}$ Human capital is more risky than physical capital, as it cannot be bought, sold or separated from its owner, whereas physical capital allows for more diversification. Our formulation of risk is different from the one in Levhari and Weiss as they assume that the state wage is a function of $\lambda$, whereas we make the likelihood of a high wage $\lambda$-dependent.

${ }^{12}$ Note that $\tilde{\alpha}>\widehat{\alpha}$ because, while for type $\widehat{\alpha}$ a marginal investment in education has no impact on wage risk, it increases the gap in utility between the high and the low states. In the limiting case with no risk aversion, $\tilde{\alpha}$ and $\widehat{\alpha}$ would coincide.
} 


\subsection{Numerical example}

Let $w_{0}=0.5, w_{L}=1$ and $w_{H}=1.5$. Let the success probability be isoelastic in investment and multiplicative in ability - a common specification in the literature $-\pi^{H}(\lambda, \alpha)=\alpha \beta_{0} \lambda^{\beta_{1}}$, with $\beta_{0}=1.5$ and $\beta_{1}=0.5$. The ability space is $A=[0.5,0.75]$. Let the quadratic utility be $v(c)=$ $1.5 c-0.25 c^{2}$, where $c$ is consumption. ${ }^{13}$ Figure 1 highlights the benchmark case using solid lines. The top panel shows how investment increases with ability. Ability types below $\widehat{\alpha}=0.62$ have a success probability below $1 / 2$. The bottom panel shows that the implicit rate of return on education, $\rho^{*}(\alpha)$, while strictly decreasing in ability, deviates from the return on savings (unity) by a modest degree for all ability types. Consider then the impact of disappointment aversion (here set at $\psi=0.2$ ), as indicated by the hatched lines. Ability types below $\widetilde{\alpha}=0.64$ reduce their investment relative to the benchmark case, while higher types increase their investment. The differential impact of disappointment by ability is reflected by the significantly more sloped gradient in the implicit rate of return on education, with lower ability types having $\rho^{*}(\alpha)$ well above unity and higher types having $\rho^{*}(\alpha)$ well below unity.
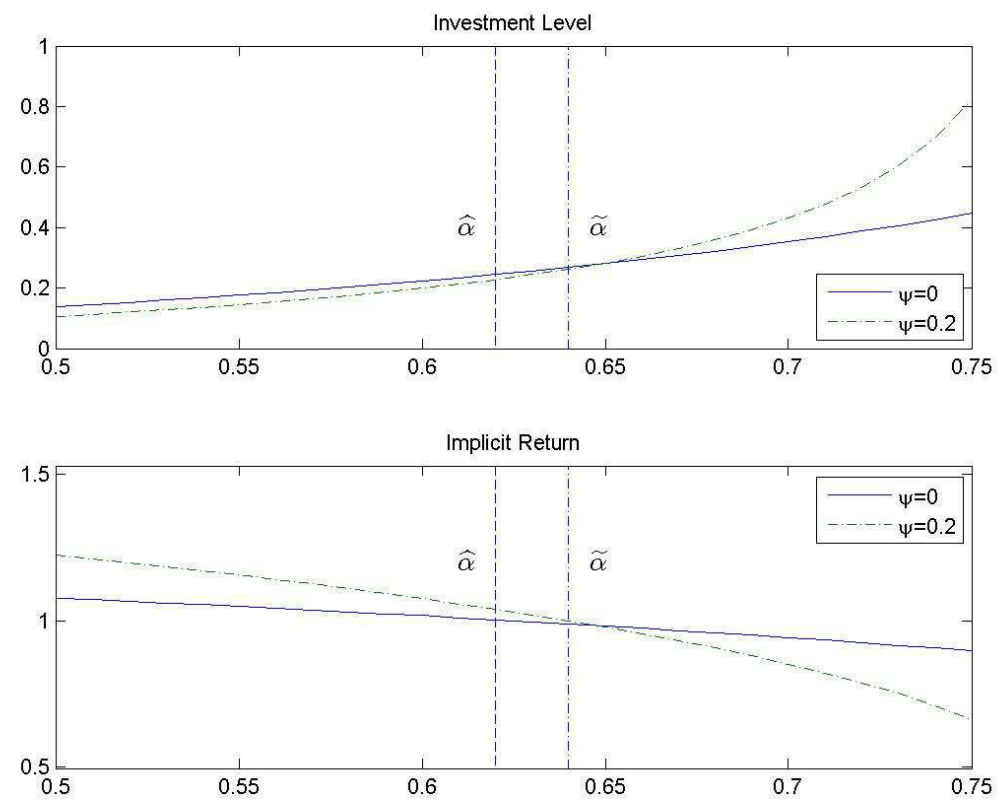

Figure 1: Investment and implicit return in the benchmark case $(\psi=0)$ and under disappointment $(\psi=0.2)$.

\section{Discussion}

The current paper has shown how disappointment aversion can generate an increased dispersion of educational investments and hence equilibrium rates of return. In this section, we briefly discuss information, choice of the reference point, welfare criterion, and policy.

We have assumed that agents are perfectly informed about their individual abilities prior to choosing educational investments. This may or may not be realistic. Consider the opposite

\footnotetext{
${ }^{13}$ Being quadratic, $v(c)$ does not satisfy $v^{\prime}>0$ globally. However, it does so within the relevant range.
} 
extreme case where each agent only knows that she has some ability $\alpha$ drawn from a distribution with pdf $f(\alpha)$. In this case, the agents are identical when making their investment choice and face a compound uncertainty over both $\alpha$ and the future wage. The same logic as above continues to apply to the commonly chosen investment $\lambda^{*}$, but with expectations taken over both $\alpha$ and the adult wage. In particular, define $\bar{\pi}^{j}(\lambda) \equiv \int_{\alpha \in A} \pi^{j}(\lambda, \alpha) f(\alpha) d \alpha$ and $\sigma(\lambda)=\bar{\pi}^{L}(\lambda) \bar{\pi}^{H}(\lambda)$. It then follows that, under disappointment aversion, each agent maximizes $V(\lambda)=\sum_{j=L, H} \bar{\pi}^{j}(\lambda) v_{j}-\psi \sigma(\lambda) \Delta v$. As a result, disappointment aversion decreases the common investment if $\bar{\pi}^{H}\left(\lambda^{*}\right)<1 / 2$ and increases it if the opposite applies. That is, the intuition behind our result does not change in this case. In the intermediate cases in which agents are "imperfectly" informed about their abilities, it is hard to draw general conclusions, as "imperfect" information can be modelled in several alternative ways and the conclusion will depend on the exact formulation.

While both lab and field evidence show that reference points are determined by expectations (Ericson \& Fuster 2011; Bartling et al. 2015), as assumed in this paper, it is interesting to note how our result would change under an alternative reference point considered in previous literature: the best outcome. If we assume the agent's reference point to be the highest wage, the discouragement effect described above no longer occurs: the investment in education increases for any ability level. The intuition is that individuals of any ability level want to reduce the probability of a low wage (particularly higher ability individuals).

It is tempting to say that disappointment aversion leads low (high) ability individuals to "underinvest" ("overinvest") in education. However, this assumes that the individuals are maximizing the "wrong" preferences, such that their chosen behaviour is not in their own long-term interest. As is well-known, loss aversion (of which disappointment aversion is a special case) generates an "endowment effect" that is not compatible with the standard notion of rationality. Due to a kink in the utility at the endowment point, an agent may prefer $x$ to $y$ when holding $x$, but $y$ to $x$ when holding $y$. It is still an open question how welfare should be evaluated in settings where agents hold loss averse preferences, as well as other non-standard preferences (see e.g. Bernheim \& Rangel 2005 and Munro 2009). While the literature has not yet reached a consensus concerning the appropriate welfare criterion, there are two main schools of thought. One view is that non-standard preferences, such as reference-dependent preferences, represent a temporary "error" and should therefore be ignored when assessing welfare. If one follows this view, this paper's analysis would indeed suggest that disappointment aversion downward (upward) biases the educational investments by low (high) ability individuals. The other view argues that if non-standard preferences are "true" and stable, they should be accounted for in the welfare criterion.

Closely related to this is the issue of policy. Only if one believes that disappointment aversion does not reflect the individuals' long-term preferences will there be a case for intervention. In an early and longer working paper version of the current paper (Anderberg \& Cerrone 2014) we generalize the model to include a labour-leisure choice in the second period and study how tax-subsidy policy can be welfare improving under the assumption that welfare is given by the individuals' expected utilities. In that case, there is a strong case for a policy that encourages educational investment, particularly at the lower end of the ability distribution. 


\section{Appendix}

\section{Proof of Proposition 1}

When $\psi=0$, the individual chooses $\lambda^{*}(\alpha)$ to maximize (1), leading to the first order condition

$$
\pi_{\lambda}^{H}\left(\lambda^{*}(\alpha), \alpha\right) \Delta v=w_{0} E\left[v^{\prime}\right]
$$

Using the assumption that $v^{\prime \prime \prime}=0$, it is easy to show that

$$
\Delta v=\frac{\left[v^{H^{\prime}}+v^{L^{\prime}}\right]}{2} \Delta w .
$$

Combining the above with the rate of return on educational investment in (5) yields

$$
\rho^{*}=\frac{\pi_{\lambda}^{H}\left(\lambda^{*}(\alpha), \alpha\right)}{w_{0}} \frac{\Delta v}{\left[v^{H^{\prime}}+v^{L^{\prime}}\right] / 2}=\frac{E\left[v^{\prime}\right]}{\left[v^{H^{\prime}}+v^{L^{\prime}}\right] / 2},
$$

where the first equality uses (A2) and the second equality uses (A1). It follows that $\rho^{*}>1$ if and only if $E\left[v^{\prime}\right]>\left[v^{H^{\prime}}+v^{L^{\prime}}\right] / 2$. Given that $v^{H^{\prime}}<v^{L^{\prime}}$, this will be the case for any agent for whom $\pi^{H}\left(\lambda^{*}(\alpha), \alpha\right)<1 / 2$. Conversely, $\rho^{*}<1$ for any agent for whom $\pi^{H}\left(\lambda^{*}(\alpha), \alpha\right)>1 / 2$.

\section{Proof of Proposition 2}

By comparative statics, $\partial \lambda^{*}(\alpha) / \partial \psi$ has the same sign as the cross-partial derivative

$$
V_{\lambda \psi}=-\sigma_{\lambda}\left(\lambda^{*}(\alpha), \alpha\right) \Delta v+w_{0} \sigma\left(\lambda^{*}(\alpha), \alpha\right) \Delta v^{\prime}
$$

Note first that $\Delta v^{\prime}<0$, whereby the second term is non-positive. Moreover, noting that

$$
\sigma_{\lambda}(\lambda, \alpha)=\pi_{\lambda}^{H}(\lambda, \alpha)\left[1-2 \pi^{H}(\lambda, \alpha)\right]
$$

it follows that $\sigma_{\lambda}\left(\lambda^{*}(\alpha), \alpha\right)>0$ for any ability type $\alpha \leq \widehat{\alpha}$. Hence for all such low ability types, disappointment aversion strictly reduces the privately optimal investment. For any ability type $\alpha>\widehat{\alpha}, \sigma_{\lambda}\left(\lambda^{*}(\alpha), \alpha\right)<0$, whereby $V_{\lambda \psi}$ may be positive.

Consider the case of limited risk aversion, that is where $v^{\prime}$ is close to constant. In the limiting case where there is no risk aversion, $v^{\prime}$ is constant and hence $\Delta v^{\prime}=0$. From (A4) we then see that, in the limit, $V_{\lambda \psi}=0$ for type $\widehat{\alpha}$. Hence in the limiting case $\widetilde{\alpha}=\widehat{\alpha}$. Outside the limit, if $\Delta v^{\prime}$ is negligible, it follows that $V_{\lambda \psi \alpha}=-\left(\sigma_{\lambda \lambda} \lambda^{\prime}+\sigma_{\lambda \alpha}\right) \Delta v>0$ for all types $\alpha>\widehat{\alpha}$, where we used that $\sigma_{\lambda \alpha}=\pi_{\alpha \lambda}^{H}\left(1-2 \pi^{H}\right)-2 \pi_{\lambda}^{H} \pi_{\alpha}^{H}<0$. This ensures that, for positive but limited risk aversion, $\widetilde{\alpha}>\widehat{\alpha}$ exists and is unique, and is close to $\widehat{\alpha}$.

\section{References}

Anderberg, D. \& Cerrone, C. (2014), 'Education, disappointment and optimal policy', CESifo Working Paper No 5141.

Bartling, B., Brandes, L. \& Schunk, D. (2015), 'Expectations as reference points: Field evidence from professional soccer', Management Science 61, 2646-2661. 
Bell, D. E. (1985), 'Disappointment in decision making under uncertainty', Operations Research 33(1), 1-27.

Bernheim, B. D. \& Rangel, A. (2005), 'Behavioral public economics: Welfare and policy analysis with non-standard decision-makers', NBER Working Papers 11518, National Bureau of Economic Research.

Cadena, B. C. \& Keys, B. J. (2015), 'Human capital and the lifetime costs of impatience', American Economic Journal: Economic Policy 7, 126-153.

Carneiro, P. \& Heckman, J. (2002), 'The evidence on credit constraints in post-secondary schooling', Economic Journal 112(482), 705-773.

Chen, S. (2008), 'Estimating the variance of wages in the presence of selection and unobserved heterogeneity', Review of Economics and Statistics 90(2), 275-289.

Ericson, K. M. M. \& Fuster, A. (2011), 'Expectations as endowments: evidence on referencedependent preferences from exchange and valuation experiments', Quarterly Journal of Economics 126, 1879-1907.

Goux, D., Gurgand, M. \& Maurin, E. (2014), 'Adjusting your dreams? The effect of school and peers on dropout behaviour', IZA Discussion Paper N. 7948.

Jensen, R. (2010), 'The (perceived) returns to education and the demand for schooling', The Quarterly Journal of Economics 125(2), 515-548.

Koszegi, B. \& Rabin, M. (2006), 'A model of reference-dependent preferences', Quarterly Journal of Economics 121(4), 1133-1165.

Koszegi, B. \& Rabin, M. (2007), 'Reference-dependent risk attitudes', American Economic Review $\mathbf{9 7}(4), 1047-1073$.

Lavecchia, A. M., Liu, H. \& Oreopoulos, P. (2014), 'Behavioral economics of education: Progress and possibilities', NBER Working Paper No. 20609.

Lecouteux, G. \& Moulin, L. (2015), 'To gain or not to lose? Tuition fees for loss averse students', Economics Bulletin 35, 1005-1019.

Levhari, D. \& Weiss, Y. (1974), 'The effect of risk on the investment in human capital', American Economic Review 64, 950-963.

Loomes, G. \& Sugden, R. (1986), 'Disappointment and dynamic consistency in choice under uncertainty', Review of Economic Studies 53(2), 271-282.

Munro, A. (2009), Bounded Rationality and Public Policy - A Perspective from Behavioural Economics, Springer Verlag.

Oreopoulos, P. \& Salvanes, K. (2011), 'Priceless: The nonpecuniary benefits of schooling', Journal of Economic Perspectives 25, 159-184. 